\title{
Investigation of Optimal Control Allocation for Gust Load Alleviation in Flight Control
}

\author{
Susan A. Frost ${ }^{1}$ \\ NASA Ames Research Center, Moffett Field, CA, 94035 \\ Brian R. Taylor ${ }^{2}$ \\ NASA Dryden Flight Research Center, Edwards, CA, 9352 \\ and \\ Marc Bodson ${ }^{3}$ \\ University of Utah, Salt Lake City, UT 84112
}

\begin{abstract}
Advances in sensors and avionics computation power suggest real-time structural load measurements could be used in flight control systems for improved safety and performance. A conventional transport flight control system determines the moments necessary to meet the pilot's command, while rejecting disturbances and maintaining stability of the aircraft. Control allocation is the problem of converting these desired moments into control effector commands. In this paper, a framework is proposed to incorporate real-time structural load feedback and structural load constraints in the control allocator. Constrained optimal control allocation can be used to achieve desired moments without exceeding specified limits on monitored load points. Minimization of structural loads by the control allocator is used to alleviate gust loads. The framework to incorporate structural loads in the flight control system and an optimal control allocation algorithm will be described and then demonstrated on a nonlinear simulation of a generic transport aircraft with flight dynamics and static structural loads.
\end{abstract}

\section{Introduction}

$\mathrm{R}$ educing the environmental impact of civil aviation and increasing safety is a goal of the NASA Aeronautics Research Directorate ${ }^{1}$. NASA, industry, universities, and other government organizations are researching advanced technologies and exploring novel civil transport configurations to achieve these goals. Environmental impact of aviation, in the form of fuel burn and emissions, will be addressed, in part, by decreasing the weight of aircraft. Advanced materials and reduced structural material decreases the empty vehicle weight, but this comes with challenges created by increased airframe flexibility and vulnerability to exceedance loads. Safe operation of these new vehicles is an important area of research.

Conventional aircraft have a flight control system that follows the pilot's commands, while maintaining stability of the aircraft and rejecting disturbances such as gusts. The flight control system determines the three angular rates in the aircraft body axis that will achieve the desired objectives. Control allocation is the problem of determining aircraft control surface deflections to achieve desired rates. Conventional control allocation schemes control the three angular rates primarily with three control variables. Control allocation on NextGen aircraft will control these rates using a variety of redundant and multi-objective control surfaces. We say a vehicle is over-actuated if it has more control effectors than control variables. The control allocation of over-actuated vehicles has been formulated as a constrained optimization problem by many researchers ${ }^{2-12}$. A real-time solution to the control allocation problem is desirable to enable the system to run on NextGen aircraft during flight. This has prompted the search for numerical optimization methods that have good convergence properties and acceptable computational requirements.

\footnotetext{
${ }^{1}$ Computer Engineer, Intelligent Systems Division, M/S 269-3, AIAA Member.

${ }^{2}$ Aerospace Engineer, Controls and Dynamics Branch, PO Box 273, M/S 4840D, AIAA Member.

${ }^{3}$ Professor, Electrical and Computer Engineering, 50 S Central Campus Dr. Rm. 3280, AIAA Senior Member.
} 
We propose a framework to enable a flight control system with optimal control allocation to incorporate realtime structural load feedback and structural load constraints in addition to effector position constraints. Structural loads are minimized in such a way to provide gust load alleviation. The following schematics show how the control allocation module fits into a flight control system and how structural load feedback can be incorporated in the control system, see figs. 1-2. In the following figures, $r$ is the reference command, $v$ is the virtual command, $u$ is the control input, $y$ is the sensor output, $x$ is the state feedback, and $M$ is the measured loads.

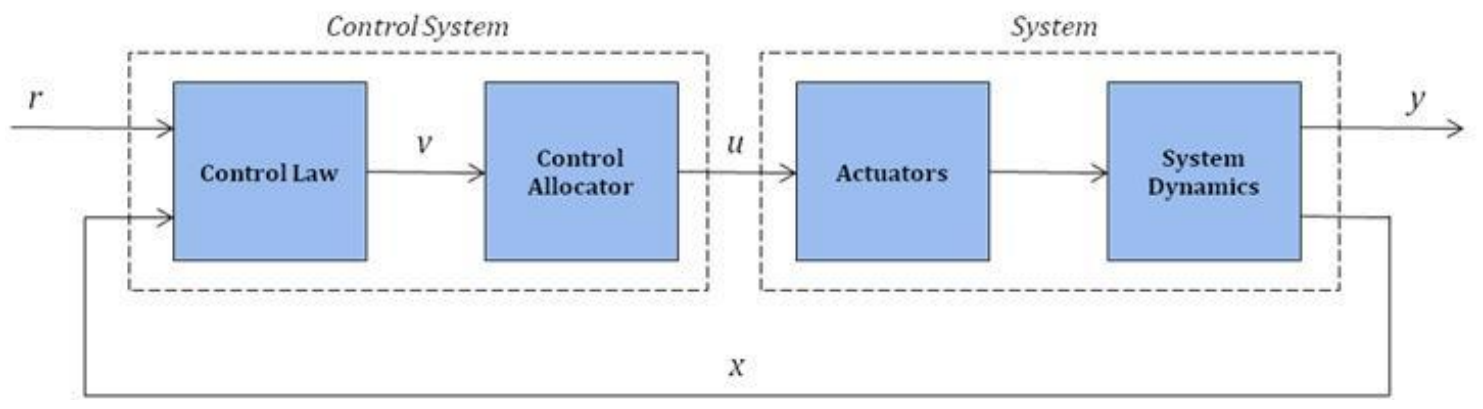

Fig. 1. Flight control system with optimal control allocation.

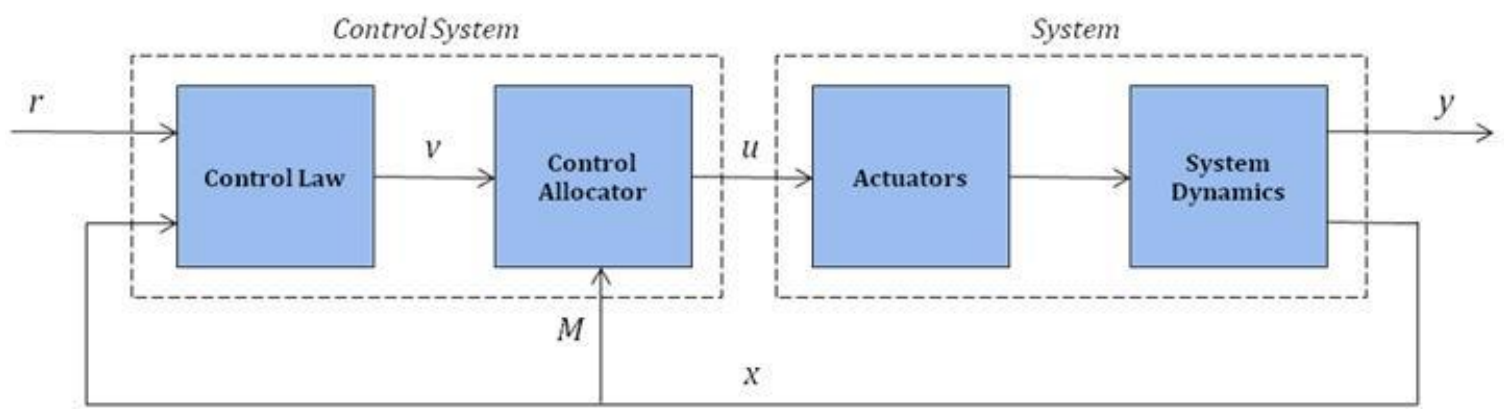

Fig. 2. Flight control system with optimal control allocation and load feedback.

An optimal control allocation algorithm that incorporates structural load measurements with load and actuator constraints will be described and demonstrated on an illustrative example. The proposed framework and control allocation algorithm are evaluated in a simulation of a generic transport aircraft coupled with a structural model of the aircraft to simulate real-time structural loads. The coupled model is used to estimate structural loads on the aircraft during flight and to predict loads generated by control surface deflections. The simulation demonstrates how optimal control allocation with load feedback and load minimization could enable aircraft to reduce aircraft structural loads during a gust encounter.

\section{Control Allocation}

Several methods to solve the control allocation problem have been evaluated, including direct allocation, linear programming, quadratic programming, and mixed optimization approaches ${ }^{2-12}$. There are advantages and disadvantages to all of the approaches. Control allocation research has also extended the control solution to include coupling or interaction effects between control effectors, creating a nonlinear optimization problem that can often be transformed into a sequence of linear problems ${ }^{10}$. While the interaction effects will be important to study for control allocation in next generation aircraft, this paper will focus on solutions that assume a linear relationship between the effectors and the moments they generate.

\section{A. Control allocation in model reference control}

We introduce control allocation in the context of model reference control (a form of dynamic inversion). However, solutions may be used in a variety of control design methods. To state the problem mathematically, we consider the state-space model 


$$
\begin{aligned}
& \dot{x}_{A}=A_{A} x_{A}+B u+d \\
& y_{A}=C x_{A}
\end{aligned}
$$

where $x_{A} \in \mathrm{R}^{\mathrm{n}}, d \in \mathrm{R}^{\mathrm{n}}, u \in \mathrm{R}^{\mathrm{p}}, y_{A} \in \mathrm{R}^{\mathrm{q}}$. For the control of aircraft, the states are given by the vector $x_{A}$ and may include the angle of attack, the pitch rate, the angle of sideslip, the roll rate, and the yaw rate $(n=5)$. The output vector $y_{A}$ may contain the pitch rate, the roll rate, and the yaw rate $(q=3)$. The control input vector $u$ consists of the commanded actuator positions. In a conventional aircraft, these commands are the deflections of two elevators, two ailerons, and the rudder $(p=5)$. The disturbance vector $d$ represents the forces and moments that the control surfaces must cancel in order to trim the aircraft (i.e., to create an equilibrium of the dynamical system).

For the purpose of example, consider a simple model reference control law. The method relies on a reference model that represents the desired dynamics of the closed-loop system

$$
\dot{y}_{M}=A_{M} y_{M}+B_{M} r_{M}
$$

where $r_{M}$ is a reference input vector (the pilot commands) and $y_{M}$ represents the desired output of the system. Since the derivative of $y$ is given by

$$
\dot{y}_{A}=C A_{A} x_{A}+C B u+C d
$$

the objective may be achieved by setting

$$
C B u=-C A_{A} x_{A}-C d+A_{M} y_{A}+B_{M} r_{M} \triangleq a_{d}
$$

where $a_{d}$ represents the desired vector to be matched by $C B u$. If $y$ is a vector composed of the incremental rotational rates (as is typically the case), $a_{d}$ represents the desired incremental rotational accelerations, and $u$ represents the incremental surface deflections.

Obtaining $u$ from $a_{d}$ requires that one solve a system of linear equations with more unknowns than equations. Solving such a system is easy, but the difficulty in control allocation is that the vector $u$ is constrained. The limits generally have the form

$$
u_{\min , i} \leq u_{i} \leq u_{\max , i} \quad \text { for } i=1, \ldots, p
$$

where $p$ is the number of surfaces. In vector form, Eq. 5 is written as $u_{\min } \leq u \leq u_{\max }$. There may be additional constraints due to the maximum rate of deflection of the actuators that can be incorporated in the minimization problem. We refer to the problem of finding a vector $u$ that is the "best" possible solution of Eq. 4 within the constraints Eq. 5 as the control allocation problem.

Given the constraints, the control allocation problem may be such that:

- many solutions exist,

- only one solution exists,

- no exact solution exists.

One is naturally drawn to finding solutions that minimize the error $C B u-a_{d}$. Indeed, providing all the control authority available may make the difference between a maneuver being achievable or not, and between an unusual condition being recoverable from or not. However, the question also arises as to which solution is the most desirable when many solutions exist. Therefore, optimal control allocation typically consists both of error minimization and control optimization. As we will discuss in this paper, the objective of load minimization, or at least load limiting, may also become part of the control allocation problem.

\section{B. Formulations of optimal control allocation}

The fundamental control allocation problem can be formulated as the following error minimization objective.

Error minimization: given a matrix $C B$, find a vector $u$ such that the cost function 


$$
J=\left\|C B u-a_{d}\right\|
$$

is minimized, subject to $u_{\min } \leq u \leq u_{\max }$.

The problem is solved exactly if $J=0$. However, regardless of whether an exact solution exists, the following control minimization problem may be considered as well.

Control minimization: given a matrix $C B$, a vector $u_{p}$, and a solution vector $u_{1}$ such that $u_{\min } \leq u_{1} \leq u_{\max }$, find a vector $u$ such that

$$
J=\left\|u-u_{p}\right\|
$$

is minimized, subject to

$$
(C B) u=(C B) u_{1}
$$

and $u_{\min } \leq u \leq u_{\max }$.

The control minimization problem is a secondary optimization objective to be satisfied if the solution of the primary objective, given by $u_{1}$, is not unique. The vector $u_{p}$ represents some preferred position of the actuators (e.g., one that yields zero deflections of the surfaces). After a solution yielding minimum error is obtained, the solution with minimum deviation from the preferred position is picked among all equivalent solutions. For both problems, weighting of the elements of the vectors may be inserted in the norms, either to prioritize the axes or to prioritize the actuators.

The norm used in the optimization criteria is a design choice that has more consequences than might be expected. The $l_{l}$ norm of a vector $x$ is the sum of the absolute values of the elements of the vector

$$
\|x\|_{1}=\sum_{i=1}^{n}\left|x_{i}\right|
$$

while the $l_{2}$ norm is the usual Euclidean norm

$$
\|x\|_{2}=\sqrt{\sum_{i=1}^{n}\left|x_{i}\right|^{2}}
$$

and the $l_{\infty}$ norm is the sup norm

$$
\|x\|_{\infty}=\max _{i}\left|x_{i}\right|
$$

A possible implementation of optimization for control allocation consists in the sequential minimization of the error vector and of the control vector. Specifically, the error is minimized first, and then the control vector is minimized among all equivalent solutions. In Ref. 5, the control minimization problem was solved only when the solution of the primary error minimization problem was $J=0$. However, it should be noted that, unless the matrix $C B$ satisfies specific conditions (any $q \times q$ submatrix of $C B$ must be nonsingular), the solution is not necessarily unique, even if the desired vector $a_{d}$ is not feasible. Given this fact, mixed optimization makes sense, and has several advantages over sequential optimization.

Mixed optimization: Given a matrix $C B$ and a vector $u_{p}$, find a vector $u$ such that

$$
J=\left\|C B u-a_{d}\right\|+\varepsilon\left\|u-u_{p}\right\|
$$


is minimized, subject to $u_{\min } \leq u \leq u_{\max }$ with $\varepsilon>0$.

The mixed optimization problem combines the error and control minimization problems into a single problem through the use of a small parameter $\varepsilon>0$. For epsilon small, priority is given to error minimization over control minimization, as is normally desired. Often, the combined problem may be solved faster, and with better numerical properties, than when the error and control minimization problems are solved sequentially ${ }^{2}$. It is possible to include a vector of weights inside the norms of Eq. 12, thereby allowing certain axes or control surfaces to be weighted differently in the cost function.

\section{Implementation of optimal control allocation algorithms}

Computational resources available on modern aircraft make the use of optimal control allocation algorithms feasible in real-time. An efficient algorithm to solve the mixed optimization problem given in Eq. 12 with the $l_{l}$ norm on the criterion was formulated by Bodson using linear programming approaches, providing guaranteed convergence to a solution in an acceptable period of time ${ }^{2}$. Timing data showed that solutions of the problem could comfortably be performed in real-time, even for large numbers of actuators, and that the optimal solution improved performance significantly over simpler, approximate methods. The algorithm was based on the revised simplex method ${ }^{13}$ with additional refinements, such as anticycling, as described in detail in Ref. 2.

Harkegård proposed an elegant solution of the optimal control allocation problem using the $l_{2}$ norm and the theory of active sets ${ }^{9}$. The algorithm was very similar to the simplex algorithm used for $l_{l}$ optimization, and had the same advantage of completing in finite time and with a small number of iterations.

Reference 11 describes and compares several new algorithms that use different combinations of norms on the optimization criteria. A particularly promising algorithm uses the $l_{l}$ norm for error minimization and the $l_{\infty}$ norm for control minimization, with both criteria combined in a single, mixed optimization criterion. The min-max criterion results in a type of resource-balancing, where the resources are the control surface deflections and the algorithm balances those resources to achieve the desired command. A small modification to the approach used in Ref. 2 for mixed $l_{l}$ optimization yields the desired linear program.

A further modification to the algorithm using the $l_{\infty}$ norm for control minimization yields the solution of a new problem where the actuator deflections are weighted in the computation of the $l_{\infty}$ norm as per unit values, where a unit is the maximum deflection of the actuator ${ }^{12}$. In this algorithm, minimization of the control effort translates into minimization of the maximum actuator deflection as a percentage of its range of motion. Advantages of the resource-balancing feature were shown to include a greater resilience to actuator failures and to nonlinear effectiveness for large actuator deflections.

\section{Control Allocation with Structural Load Constraints and Load Feedback}

Most optimal control allocation algorithms find an optimal solution to the control allocation problem within the constraints of the control surface position and possibly rate limits. However, these constraints are not sufficient to ensure that the structural load limits of the aircraft will not be exceeded by the commanded control surface deflections. The bending and torsion moments at the wing root are examples of loads on the aircraft that need to be monitored. In this section, we formulate the load constraints at discrete critical points on the aircraft as

$$
|M+T u| \leq L_{\max }
$$

where $M$ is a vector of the current measured or estimated loads at the critical points, $T$ is a matrix that converts the effect of incremental surface deflections into incremental structural loads, and $L_{\max }$ is a vector of maximum allowable structural loads at the critical points. The loads that need to be limited are a function of the aircraft being considered, often with an emphasis on torsion and bending moments and shear forces. Generally, the load limits are determined through detailed studies, including ground and flight tests. This paper will not address the selection of the location or the number of load points to be considered for a given problem. For the purpose of developing a representative example, we choose load points along the aircraft wing, horizontal tail, and vertical tail.

We assume that the $T$ matrix, which is computed from the states of the aircraft at the current time, gives a linear approximation of the incremental structural loads arising from commanded surface deflections. The incremental loads matrix is formed by perturbing each control surface deflection from its current position at the current aircraft state. The perturbation yields the change in aerodynamic lift and rolling moments due to a one-degree change in surface deflection. It is assumed that the lift due to the wings is elliptically distributed along the span of each wing. 
It is also assumed that the control effectiveness of each surface is proportional to the lift generated by that control surface. The resulting lift and moment components are used in conjunction with a structural model of the aircraft to determine moments at critical points on the aircraft. Superposition of the control surface effects in terms of lift, moments, and structural loads is assumed in order to obtain a reasonable, but tractable solution in real-time.

The structural load limits can be implemented as an additional constraint as given in Eq. 13. Another possibility is to include a term to minimize the loads at critical points by minimizing the following cost function

$$
J=\left\|C B u-a_{d}\right\|+\varepsilon\left\|u-u_{p}\right\|+\gamma\|M+T u\|
$$

with $\varepsilon>0$ and $\gamma \geq 0$. The choice of the weight on the load minimization is application specific. For example, to reduce the size and computation time for solving Eq. 14, load minimization might not be employed under standard operating conditions (e.g., $\gamma=0$ ). In other cases, where the load builds up due to a maneuver or gust, load minimization would be enabled by setting $\gamma>0$. Additional details on the use of weights in the cost criterion are provided in the following section.

The choice of the norm to be used for load minimization in Eq. 14 has an effect on computation time and the distribution of the loads. The $l_{\infty}$ norm could be used in a similar approach as its use for control minimization described in Section II (c) and the critical load points could be weighted in the computation of the $l_{\infty}$ norm as per unit values, where a unit is the maximum load limit of the critical point. Using this approach, solutions could be obtained that more evenly distribute loads at the critical points. Unfortunately, the computational complexity of the problem increases with each load point added, suggesting that a practical solution in which load minimization is performed only when needed to alleviate a build-up of loads from a gust or possibly a maneuver.

\section{Proof of Concept Simulation Architecture}

In this section we describe the proof of concept simulation implemented to demonstrate the proposed framework assuming static loads, an elliptical lift distribution, and a limited number of critical load points. An earlier study had a similar architecture, but only modeled the aircraft wings and used the $l_{l}$ norm. ${ }^{14}$ Use of the most effective surfaces is a recognized consequence of using the $l_{l}$ norm in control allocation. This effect was observed in the previous study, and it was seen to be a limitation. A second study was performed that used the $l_{\infty}$ norm on the control effort and demonstrated the ability of the control allocator to accommodate stuck actuators or situations where certain loads must be limited. ${ }^{15}$

\section{A. Aircraft configuration and aerodynamic model}

A full nonlinear simulation of a class of full-scale generic transport aircraft is used for the proof of concept study of the proposed framework. The simulation uses a dynamically scaled representative transport model that is derived from a model of NASA's AirSTAR testbed. The AirSTAR testbed is being developed as part of NASA's Aviation Safety Program to investigate dynamics modeling and control of large transport vehicles in upset conditions ${ }^{16}$. The AirSTAR unmanned aerial vehicle (UAV) is a 5.5\% dynamically scaled aircraft based on wind tunnel and flight test data ${ }^{17}$. The simulation used in this paper is a full-scale model that was derived from the subscale model by incorporating Reynolds adjusted aero tables, actuator models appropriate for a full-scale aircraft, and a model of NASA Glenn's Simp2 engine which is a simplified version of C-MAPSS40k.

The simulation created for this study represents a conventional modern midsize commercial passenger configuration. The aircraft has right and left inboard and outboard elevons, three ailerons on each wing, and upper and lower rudders for use by the control allocator to achieve the desired roll, pitch, and yaw moments commanded by the flight control system. A stabilizer is used for trimming the aircraft.

For the proof of concept study, seventeen critical points on the aircraft were monitored for bending moments. Each wing had critical points located at the wing root and at locations inboard of each aileron. The bending moments were monitored at locations inboard of each elevon on the horizontal tail and at the root. The vertical tail had three monitored points at the root and upper and lower rudders. The load limits were set to $\pm 14 \times 10^{6}$ for all of the critical points. These values were chosen for simplicity and to ensure that the baseline maneuver would not cause any load limiting. 


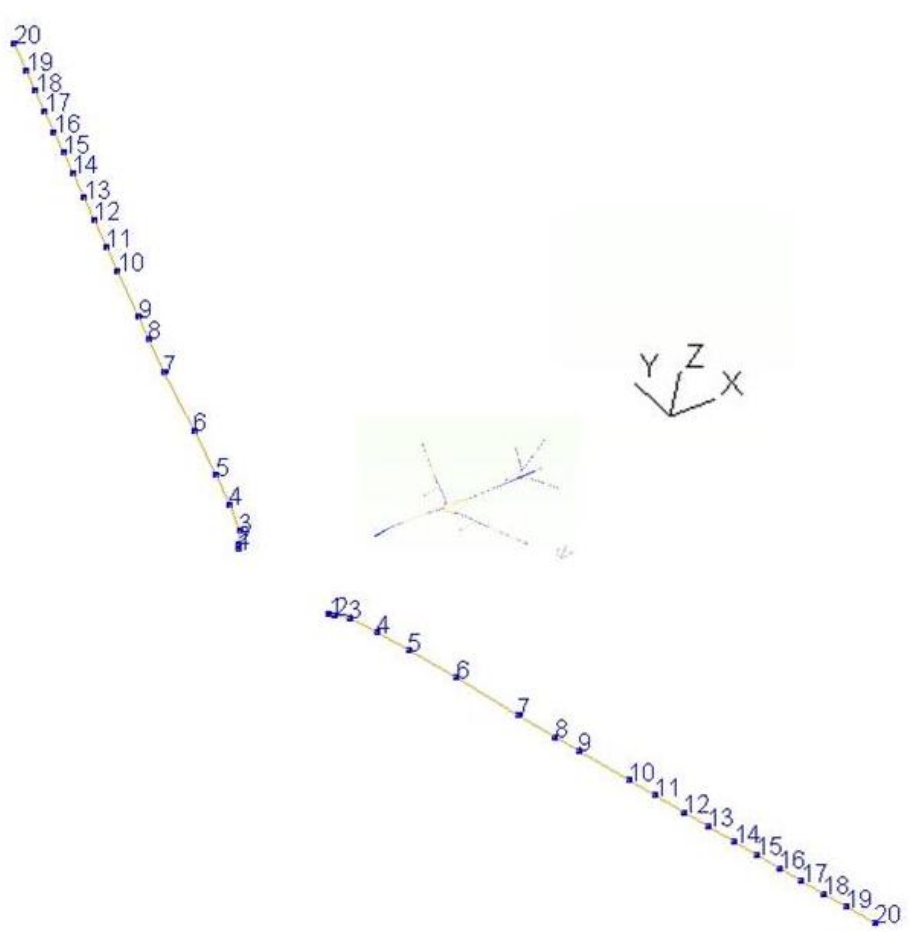

Fig. 3. Finite element beam model for left and right wings.

\section{B. Static structural model}

A finite element analysis (FEA) model is developed and integrated into the simulation to estimate static structural loads on the aircraft due to lift and roll moments and to compute the incremental loads created by surface deflections. For the proof of concept study, bending moments on the wing, tail and rudder are monitored. The finite element method is employed for its ability to calculate internal loads for potentially complex load distributions and geometries at different locations on-the-fly.

The basic FEA model requires a small number of matrix multiplications for static loads analysis. Furthermore, the finite element method provides an efficient framework for adding additional monitored points. In implementing this approach, we hope to determine whether the required computations are fast enough and accurate enough for flight controllers in both real-time simulations and actual flight.

The FEA modeling is simplified to beam modeling, based on mass and stiffness approximations for a representative full-scale generic civil transport model. The model includes two beam meshes for representing the left and right wings independently, and two beam meshes for the horizontal tails and one beam mesh for the vertical tail.

Figure 3 shows the two wing meshes with the node numbers. Each wing mesh has 20 nodes and 19 beams. The wings are modeled as cantilever beams with the fixed ends at the wing roots. All degrees of freedom for node 1 are

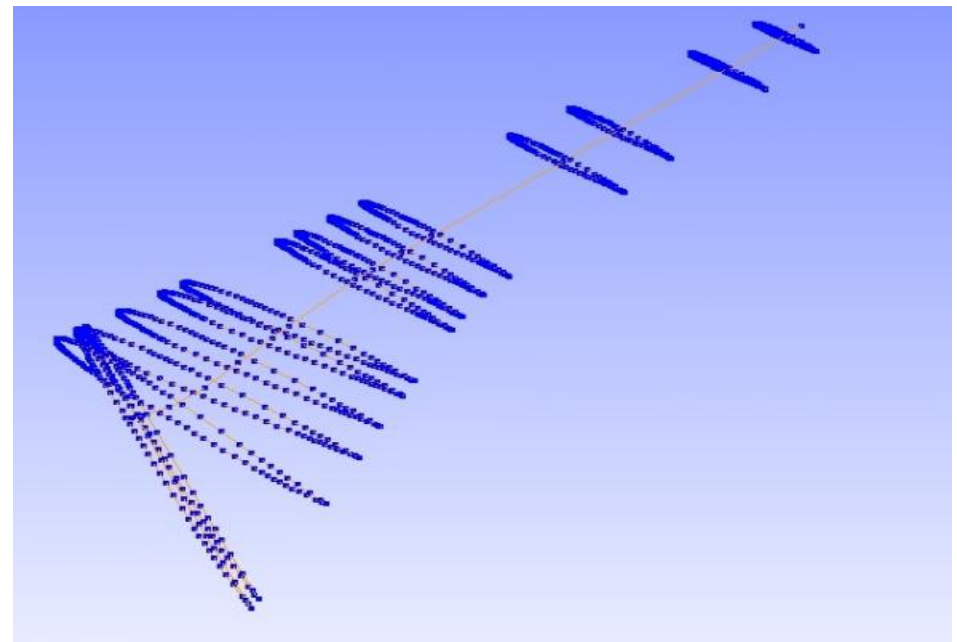

Fig. 4. Beam nodes located at centroids of wing cross sections.

fixed for both meshes. The global coordinates of the finite element model are attached to the aircraft, with the origin at the nose, positive $X$-axis pointing toward the tail, and positive $Z$-axis pointing up.

We use a simplified approximation of the wing as hollow aluminum shells having the wing outer mold line of the representative aircraft and a constant thickness of five inches. Beam cross section properties are calculated using this hollow shell wing geometry. Figure 4 shows nodes of the right wing beam mesh running through centroids of the wing cross sections.

The $\mathrm{C}^{0}$ approach as described in Ref. 18 is used for the beam modeling. There are three translation and three rotation degrees of freedom per node. A linear shape function with one-point reduced integration is used for the axial, transverse shear, torsion and transverse bending components. The "residual bending flexibility" technique $^{18}$ is applied to the shear term to improve the accuracy of the two-node, one-point quadrature beam element. The displacement solution for the static problem is given by

$$
d=K^{-1} F
$$


where $d$ is a vector containing the displacement solutions, $K$ is the stiffness matrix of the wing structure, and $F$ is a vector of external loads applied to the structure. All terms in Eq. 15 are calculated in the global coordinate system. The internal moment about local beam direction $2, M_{2}$, is the bending moment used as the measured load at the critical points passed to the control allocator in the simulation. The calculation for the internal moment for the beams is given by

$$
M_{2}=E I_{22} k_{22}
$$

where $E$ is Young's modulus, $I_{22}$ is the second moment of inertia about the local 2 beam axis, and $k_{22}$ is the curvature about the local 2 beam axis, which is calculated from the displacement solutions and the beam finite element shape function.

The structural modeling and analysis used for this study assumes static conditions and considers external loads on the wing due to aerodynamic lift forces and roll moments. The lift distribution on each wing is assumed to be elliptical, with each wing carrying half of the lift due to the wings. Figure 3 shows the normalized lift per length distribution for each wing. The external loads arising from the roll moment on the aircraft wing are assumed to be from concentrated forces applied in the aircraft $Z$ direction at the ailerons. These forces are applied to nodes 15,17 , and 19 , located at the center of the three ailerons on each wing. The $Z$ direction force acting on each aileron is assumed to be proportional to the aileron deflections. The horizontal and vertical tails are treated in a similar

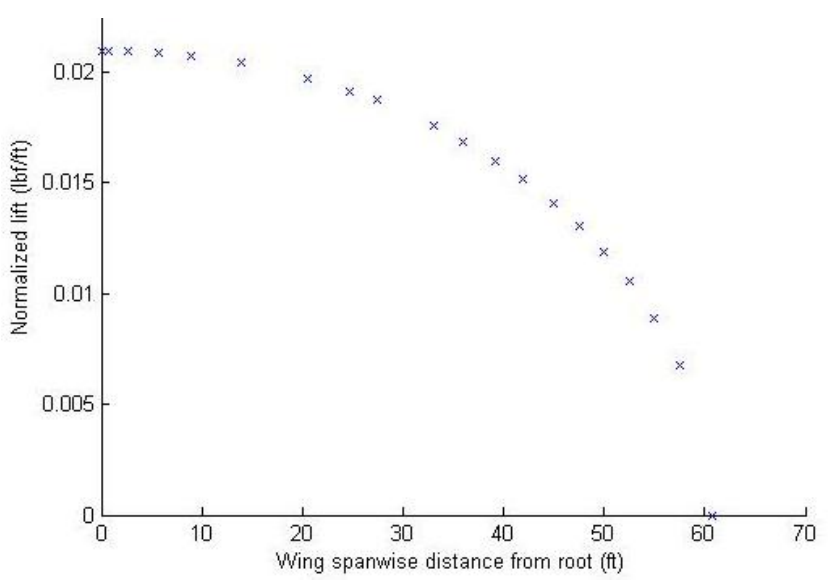

Fig. 5. Normalized lift load on each wing (lbf/ft).

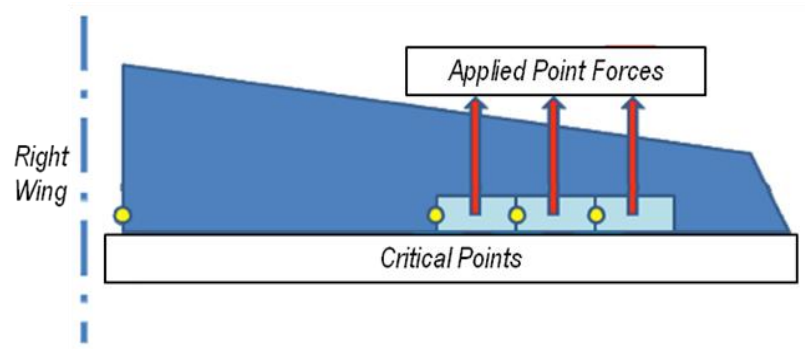

Fig. 6. Applied forces and critical points along wing. capture the rough order of magnitude dynamics of the control allocator's response to a gust, not to provide a highfidelity model of the structural response of the aircraft to maneuvering or gust encounters. A 1-cos gust model implemented according to FAR 25.341 is incorporated in the simulation described above. The gust affects the aircraft asymmetrically by impacting the middle right aileron. The gust magnitude is 50 fps in the vertical direction with a length of 25 times the chord length according to FAR 23.333. The gust $u$ and $w$ components are added to the $u$ and $w$ components of the rigid body aircraft. The angle of attack is computed from $u$ and $w$ of the rigid body plus 
gust and the rigid body without gust. The non-dimensional lift is computed from an assumed lift curve slope of $2 \pi /$ radian and the angle of attack with and without the gust. The lift is then dimensionalized by the dynamic pressure and wing area. The component of lift due to the gust is then added to the vertical force at the middle right aileron location and input into the FEM to produce the measured load resulting from the gust. The gust load build-up, without load minimization, can be seen as the solid red line in fig. 7.

\section{Stability and control augmentation system with optimal control allocation}

The stability and augmentation system for the simulation uses a dynamic inversion controller with a second order reference model. The simulation has multiple surfaces that control multiple axes. The control allocator is able to use these surfaces to achieve the desired roll, pitch, and yaw moments commanded by the flight control system. The allocator inputs are: measured loads $M$, incremental load matrix $T$ for predicting the control surface contribution to the load at the critical points, preferred position $u_{p}$, surface position limits $u_{\min }, u_{\max }$, and load limits $L_{\max }$. The rate limiting of the actuators is handled by the actuator servocontrol loop. The position and load limits are incorporated as

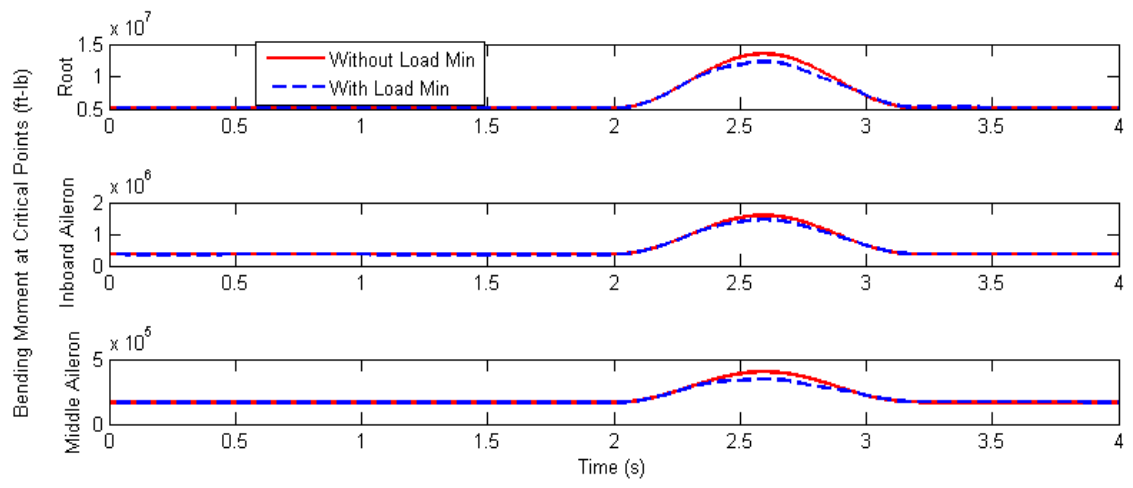

Fig. 7. Measured load on right wing critical points with and without load minimizing control allocation.

constraints on the cost function. Since this paper is looking at the feasibility of the framework, we are only considering the load constraints representing bending moments at the 17 critical points along the wings and tail. The control allocation problem solved in this simulation is to find the vector $u$ such that

$$
J=\left\|C B u-a_{d}\right\|_{1}+\varepsilon\left\|u-u_{p}\right\|_{\infty}+\|\Gamma(T u+M)\|_{1}
$$

is minimized, subject to $u_{\min } \leq u \leq u_{\max }$ and $|M+T u| \leq L_{\max }$ with $\varepsilon>0$ and $\Gamma \geq 0$. The above criterion and constraints are converted to a linear program and solved using the revised simplex algorithm described in Section II
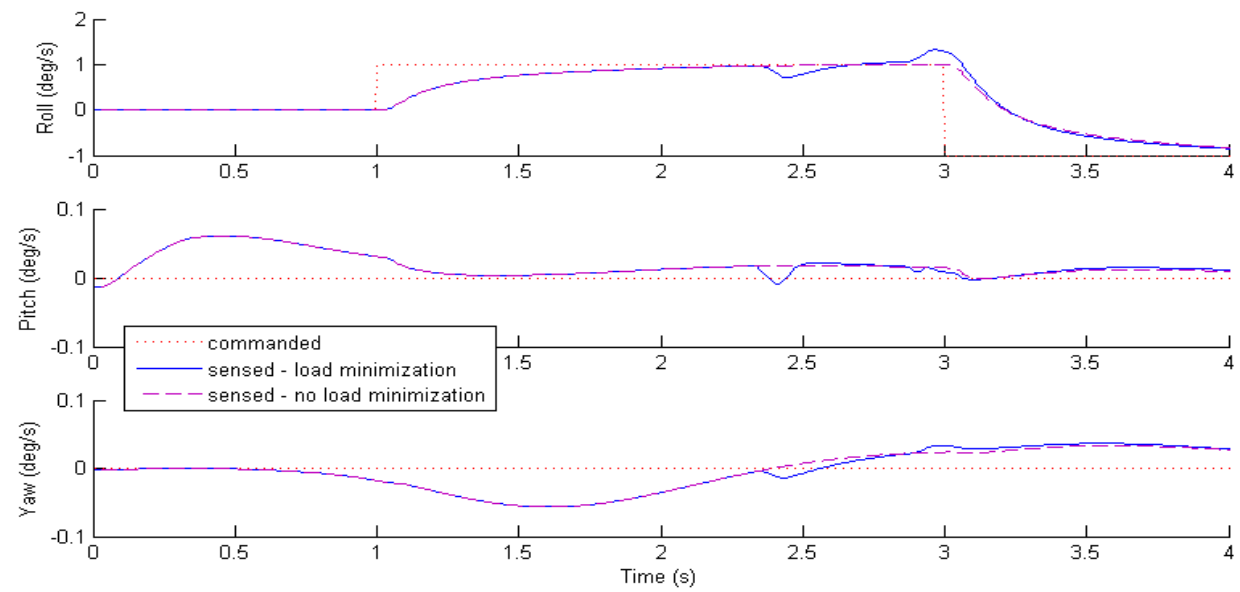

Fig. 8. Commanded and sensed rates for control allocation using load minimization and no load minimization. (c), which was modified to include the load constraints. The control allocator does not have actuator rate limits as a constraint, but the actuators modeled in the simulation rate limit their commands. The control minimization criterion was weighted in the control allocator by setting $\varepsilon=10^{-4}$. Load minimization was enabled for certain critical load points by setting values in the column vector $\Gamma$. These values were increased 
from zero to $10^{-6}$ depending on the application specific load build-up as described in the following results section. To achieve good numerical properties for the algorithm, some of the inputs to the control allocator are scaled; in particular, the elements of $C B$ and $a_{d}$ are divided by $10^{3}$ and the elements of $M, T$, and $L_{\max }$ are divided by $10^{4}$.
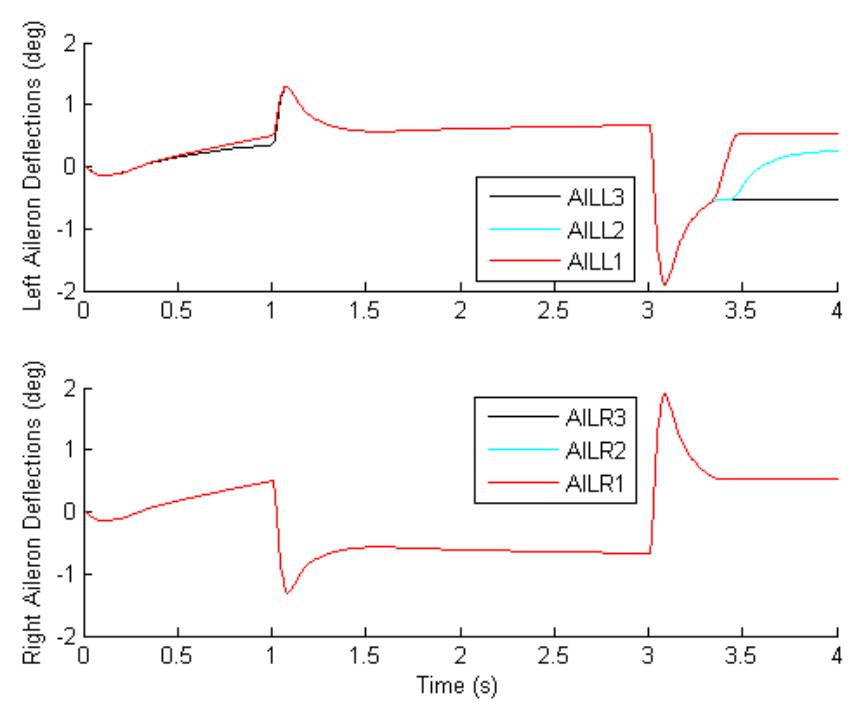

Fig. 9. Aileron deflections with control allocation with no load minimization.
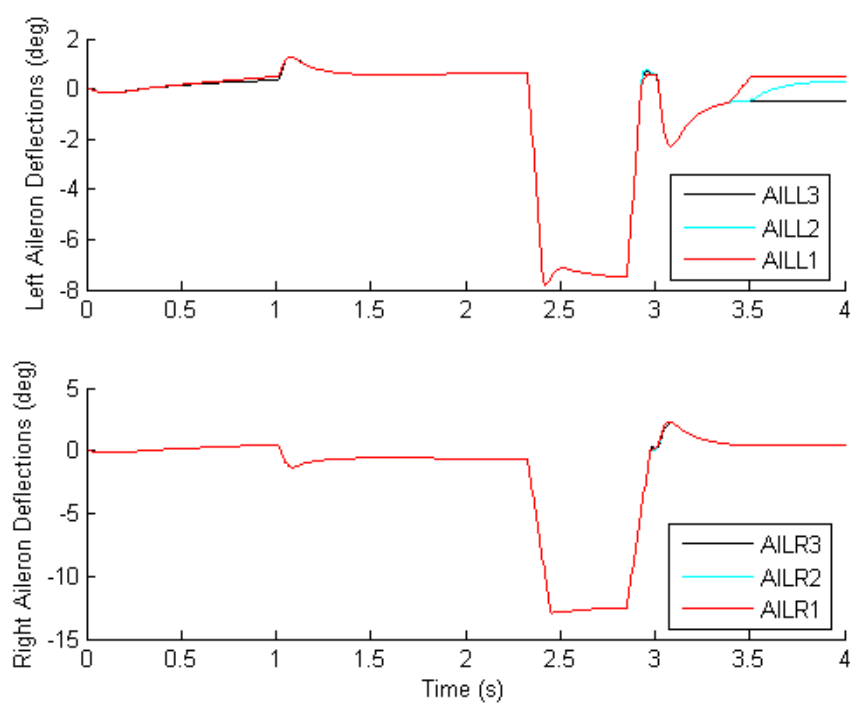

Fig. 10. Aileron deflections (deg) with load minimization.

\section{Simulation Results}

Simulations with the architecture described in Section IV were tested with a 1 -cosine gust model affecting the right wing. The simulations were run after the aircraft was trimmed at Mach 0.70 and 30,000 feet. The gust began two seconds into the simulation and lasted for one second. A baseline case without load minimizing control allocation was compared with a case with load minimizing control allocation. See fig. 7 for measured loads on right wing critical points affected by the gust for both cases. Figure 8 shows the aircraft rate responses to a roll command during the two simulation runs with the gust. Both control allocation algorithms provide acceptable command following. The control surface deflections when the control allocation had no load minimization can be seen in fig. 9 .

When the measured aircraft loads are far from the load limits and the desired moment for flight dynamics are easily achieved, the control allocator gives surface deflections that achieve the desired moment commands while moving toward the preferred surface positions $u_{p}$ that are all set to zero deflection. As the measured loads start to approach the load limits, the weighting on specific critical load points can be set in $\Gamma$ to cause the surface deflections to minimize load for those critical points while achieving the desired moment command.

In this study, the measured load as a percent of the design load limit at the critical points was monitored. When the percent load increased beyond $70 \%$, which first occurred at the right wing root due to the integration of the load along the wing, the elements of the vector $\Gamma$ corresponding to the right wing critical points were set to $10^{-6}$. This caused the load minimization criterion to be included in the cost function, thereby enabling a reduction in load while providing adequate command following, see fig. 10. The right wing ailerons were used by the control allocation algorithm to reduce the gust load, and the left ailerons were seen to deflect downward to enable the aircraft dynamic moments to be achieved. Since load alleviation required the ailerons to deflect toward their minimum position, the preferred position for the right ailerons were set to their minimum position, affecting the control minimization criterion. A simple mapping between load points and control surfaces that can significantly affect the load points determines the control surfaces that need modified preferred positions during load minimization. Once the measured load as a percent of design load limit moves below $70 \%$, the corresponding elements of the vector $\Gamma$ are set back to zeros and the original preferred positions are used. 


\section{Conclusions}

A flight control framework is proposed and demonstrated in simulation, using load constraints and real-time load feedback in conjunction with optimal control allocation. The framework was used to demonstrate how gust loads can be reduced using optimal control allocation in a simulation of a generic transport aircraft.

\section{Acknowledgments}

This work was performed under the support of the Subsonic Fixed Wing Project under NASA's Fundamental Aeronautics Program.

\section{References}

1“Fundamental Aeronautics Research Directorate", National Aeronautics and Space Administration, June 2012, URL: http://www.aeronautics.nasa.gov

${ }^{2}$ Bodson, M., "Evaluation of Optimization Methods for Control Allocation," AIAA Journal of Guidance, Control, and Dynamics, vol. 25, no. 4, pp.703-711, 2002.

${ }^{3}$ Durham, W. "Constrained Control Allocation," Journal of Guidance, Control, and Dynamics, vol. 16, no. 4, 1993, pp. 717725.

${ }^{4}$ Enns, D., "Control Allocation Approaches," AIAA Guidance, Navigation and Control Conference and Exhibit, AIAA, Boston, MA, August 1998, AIAA-1998-4109.

${ }^{5}$ Buffington, J. "Modular Control Law Design for the Innovative Control Effectors (ICE) Tailless Fighter Aircraft Configuration 101-3," Report AFRL-VA-WP-TR-1999-3057, Air Force Research Laboratory, Wright-Patterson AFB OH 454337542, 1999.

${ }^{6}$ Burken, JJ, Lu, P., Wu, Z. \& Bahm, C., "Two Reconfigurable Flight-Control Design Methods: Robust Servomechanism and Control Allocation," Journal of Guidance, Control, and Dynamics, vol. 24, no. 3, 2001, pp. 482-493.

${ }^{7}$ Petersen, J. \& Bodson, M., "Interior-Point Algorithms for Control Allocation," Journal of Guidance, Control, and Dynamics, vol. 28, no. 3, 2005, pp. 471-480.

${ }^{8}$ Petersen, J. \& Bodson, M., "Constrained Quadratic Programming Techniques for Control Allocation," IEEE Trans. on Control Systems Technology, vol. 14, no. 1, January 2006, pp. 91-98.

${ }^{9}$ Härkegård, O., "Efficient Active Set Algorithms for Solving Constrained Least Squares Problems in Aircraft Control Allocation," 41st IEEE Conference on Decision and Control, Vol. 2, pp. 1295-1300.

${ }^{10}$ Oppenheimer, M. W. \& Doman, D. B., "A Method for Including Control Effector Interactions in the Control Allocation Problem," report AFRL-VA-WP-TP-2007-309, Air Force Research Laboratory, Wright-Patterson AFB, OH 45433-7542, 2007.

${ }^{11}$ Bodson, M. \& Frost, S., "Load Balancing in Control Allocation”, Journal Guidance, Control and Dynamics, Vol. 34, No. 2, pp. 380-387, March-April 2011.

${ }^{12}$ Frost, S.A. \& Bodson, M, "Resource Balancing Control Allocation,” American Control Conference, Baltimore, MD, June 2010.

${ }^{13}$ Luenberger, D., Introduction to Linear and Nonlinear Programming, Addison-Wesley, Reading, MA 1984, pp. 80.

${ }^{14}$ Frost, S.A., Taylor, B.R. Jutte, C.V, Burken, J.J., Trinh, K.V., \& Bodson, M., "A Framework for Optimal Control Allocation with Structural Load Constraints," Proceedings AIAA Atmospheric Flight Mechanics Conference, AIAA, Toronto, ON, Canada, August 2010.

${ }^{15}$ Frost, S.A., Taylor, B.R. Jutte, C.V, Burken, J.J., Trinh, K.V., \& Bodson, M., “Application of Structural Load Feedback in Flight Control,” Proceedings AIAA Atmospheric Flight Mechanics Conference, AIAA, Toronto, ON, Canada, August 2012.

${ }^{16}$ Jordan, T.L., Foster, J.V., Bailey, R.M., \& Belcastro, C.M., "AirSTAR: A UAV Platform for Flight Dynamics and Control System Testing", AIAA 2006-3307, 25th AIAA Aerodynamic Measurement Technology and Ground Testing Conference, AIAA, San Francisco, CA, 2006.

${ }^{17}$ Jordan, T.L., Langford, W.M., and Hill, J.S., "Airborne Subscale Transport Aircraft Research Testbed, Aircraft Model Development", AIAA 2005-6432, AIAA Guidance, Navigation, and Control Conference, AIAA, Washington, DC, 2005.

${ }^{18}$ Hughes, Thomas J.R., The Finite Element Method-Linear Static and Dynamic Finite Element Analysis, Springer-Verlag, New York, 1983, Chapter 5. 